\title{
Müllerian duct anomalies with term pregnancy: a case report
}

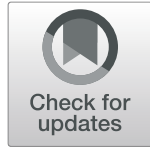

\author{
Engku Ismail Engku-Husna* ${ }^{*}$, Nik Lah Nik-Ahmad-Zuky and Kadir Muhammad-Nashriq
}

\begin{abstract}
Background: Müllerian duct anomaly is a rare condition. Many cases remain unidentified, especially if asymptomatic. Thus, it is difficult to determine the actual incidence. Müllerian duct anomaly is associated with a wide range of gynecological and obstetric complications, namely infertility, endometriosis, urinary tract anomalies, and preterm delivery. Furthermore, congenital anomalies in pregnant mothers have a high risk of being genetically transmitted to their offspring.

Case presentation: We report a case of a patient with unsuspected müllerian duct anomaly in a term pregnancy. A 33-year-old Malay woman with previously uninvestigated involuntary primary infertility for 4 years presented with acute right pyelonephritis in labor at 38 weeks of gestation. She has had multiple congenital anomalies since birth and had undergone numerous surgeries during childhood. Her range of congenital defects included hydrocephalus, for which she was put on a ventriculoperitoneal shunt; imperforated anus; and tracheoesophageal fistula with a history of multiples surgeries. In addition, she had a shorter right lower limb length with limping gait. Her physical examination revealed a transverse scar at the right hypochondrium and multiple scars at the posterior thoracic region, levels T10T12. Abdominal palpation revealed a term size uterus that was deviated to the left, with a singleton fetus in a nonengaged cephalic presentation. The cervical os was closed, but stricture bands were present on the vagina from the upper third until the fornices posteriorly. She also had multiple rectal prolapses and strictures over the rectum due to previous anorectoplasty. An emergency cesarean delivery was performed in view of the history of anorectoplasty, vaginal stricture, and infertility. Intraoperative findings showed a left unicornuate uterus with a communicating right rudimentary horn.

Conclusion: Most cases of müllerian duct anomaly remain undiagnosed due to the lack of clinical suspicion and the absence of pathognomonic clinical and radiological characteristics. Because it is associated with a wide range of gynecological and obstetric complications, it is vital for healthcare providers to be aware of its existence and the role of antenatal radiological investigations in its diagnosis. The presence of multiple congenital abnormalities and a history of infertility in a pregnant woman should warrant the exclusion of müllerian duct anomalies from the beginning. Early detection of müllerian duct anomalies can facilitate an appropriate delivery plan and improve the general obstetric outcome.
\end{abstract}

Keywords: Müllerian duct anomaly, Congenital anomaly, Imperforated anus, Unicornuate uterus, Case report

\footnotetext{
* Correspondence: drengkuhusna@gmail.com; enhusna@usm.my Department of Obstetrics \& Gynaecology, School of Medical Sciences, Universiti Sains Malaysia, Kubang Kerian, Kelantan 16150, Malaysia
}

(C) The Author(s). 2020 Open Access This article is licensed under a Creative Commons Attribution 4.0 International License, which permits use, sharing, adaptation, distribution and reproduction in any medium or format, as long as you give appropriate credit to the original author(s) and the source, provide a link to the Creative Commons licence, and indicate if changes were made. The images or other third party material in this article are included in the article's Creative Commons licence, unless indicated otherwise in a credit line to the material. If material is not included in the article's Creative Commons licence and your intended use is not permitted by statutory regulation or exceeds the permitted use, you will need to obtain permission directly from the copyright holder. To view a copy of this licence, visit http://creativecommons.org/licenses/by/4.0/. The Creative Commons Public Domain Dedication waiver (http://creativecommons.org/publicdomain/zero/1.0/) applies to the data made available in this article, unless otherwise stated in a credit line to the data. 


\section{Introduction}

Müllerian duct abnormalities (MDAs) are a broad and complex spectrum of defects that are often associated with primary amenorrhea, infertility, endometriosis, and obstetric complications. Women with congenital uterine malformation usually experience a higher incidence of complications during pregnancy and delivery. An obstetrician should have a high index of suspicion for an undiagnosed congenital defect such as MDA in the presence of infertility with a background of maternal congenital malformations. Early diagnosis and recognition of the condition may allow proper planning of treatment to ensure a favorable obstetric outcome.

\section{Case presentation}

We report a case of MDA diagnosed during an emergency cesarean section of a pregnant woman with multiple congenital anomalies. A 33-year-old Malay woman with 4 years of previously uninvestigated involuntary primary infertility had spontaneously conceived. She presented with acute right pyelonephritis in labor at 38 weeks of gestation. Of note, she has had multiple underlying congenital anomalies since birth and had undergone various surgeries during childhood. The abnormalities included hydrocephalus, for which she was put on ventriculoperitoneal shunting; an imperforated anus; and tracheoesophageal fistula with a history of multiples surgeries. In addition, she had a shorter right lower limb length with limping gait. A detailed ultrasound scan at 20 weeks of gestation revealed a grossly normal fetus. Her medication history was not significant, and her social and family history revealed no remarkable findings.

Clinically, she was alert and not in sepsis. Her vital signs were stable. However, she had a low-grade fever of $37.5^{\circ} \mathrm{C}$. She had a transverse scar on her right hypochondrium and multiple scars at the posterior thoracic region at levels T10-T12. Her abdominal examination revealed a term size uterus that was deviated to the left, with a singleton, nonengaged fetus in cephalic presentation. The result of a right renal punch was positive. Her cervix was unfavorable, with fibrotic stricture bands at the upper one-third of the vagina up to the fornices. She also had multiple rectal prolapses and strictures from a previous anorectoplasty. Her kidneys were of normal shape and size on her ultrasound examination. Her blood parameters, including her renal profile, were within normal ranges. She was administered a broadspectrum intravenous antibiotic.

In view of the above-mentioned maternal conditions, an emergency cesarean section was performed. Intraoperatively, MDA of left unicornuate uterus with fused right communicating horn was diagnosed. Both of the patient's fallopian tubes and ovaries were normal (Figs. 1 and 2). A healthy and normal baby boy weighing $2500 \mathrm{~g}$

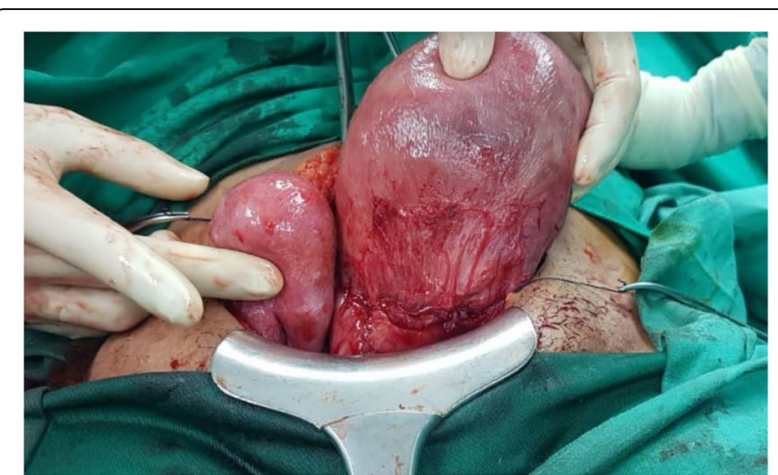

Fig. 1 Intraoperative findings illustrating the left unicornuate uterus with a communicating right rudimentary horn (anterior view). The incision of the uterine segment was on the left side

was delivered. He was vigorous at birth. Postoperatively, the intravenous antibiotic was continued for 3 days. Her condition improved, and the results of all cultures were negative. She was discharged on day 4 postoperatively and remained well at her 6-month follow-up.

\section{Discussion}

The müllerian ducts are embryologic structures that exist in pairs before undergoing a mechanism of fusion and resorption in utero to form the uterus, fallopian tubes, cervix, and upper two-thirds of the vagina. MDAs are rare conditions. The actual prevalence of MDAs is difficult to ascertain because many of the cases are asymptomatic and thus remain undiagnosed. The reported prevalence of MDAs varies widely across studies. In a systematic review, the prevalence of MDAs was $5.5 \%$ in an unselected population, $8 \%$ in infertile women, $12.3 \%$ in women with a history of miscarriage, and $24.5 \%$ in women with a history of miscarriage and infertility [1].

The American Society for Reproductive Medicine (formerly the American Fertility Society) classification system for müllerian defects has been applied as the

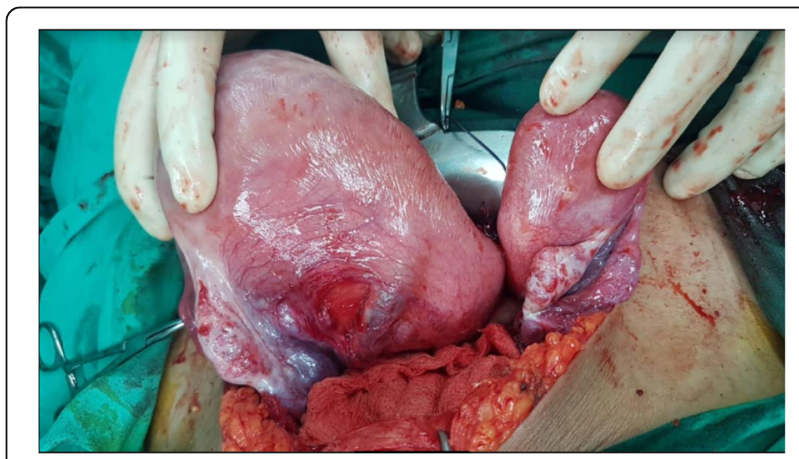

Fig. 2 Intraoperative findings revealed a left unicornuate uterus with a communicating right rudimentary horn (posterior view). The patient's fallopian tubes and ovaries were normal 
standard definition in the United States for decades [2]. When MDAs are diagnosed, any associated anomalies of the vagina, cervix, fallopian tubes, and renal system should also be identified and documented, even though they are not listed as part of the classification system. In a literature review regarding infertile and fertile women with MDAs, the frequencies of specific anomalies were septated uterus (35\%t), bicornuate uterus $(26 \%)$, arcuate uterus (18\%), unicornuate uterus (10\%), didelphys $(8 \%)$, and agenesis (3\%) [3].

MDAs are frequently associated with adverse gynecologic and obstetric complications, such as infertility, endometriosis, and miscarriage. They are also commonly associated with renal anomalies in as many as $30-50 \%$ of cases. The common renal anomalies are renal agenesis, ectopic kidney, hypoplasticity, fusion, duplication, and malrotation of the kidneys $[4,5]$. Thus, the identification of both kidneys during the investigation is essential. MDAs are also frequently seen in women born with complex anorectal malformations. Other congenital abnormalities commonly associated with MDAs include vertebral body anomalies (29\%) (that is, fused or wedged vertebral bodies, spina bifida; 22-23\%), heart abnormalities (14.5\%), and syndromes such as the Klippel-Feil syndrome (7\%) [6]. Other conditions involving vertebral defects, anal atresia, cardiac defects, tracheoesophageal fistula, renal anomalies, and limb abnormalities (VACT ERL association), such as renal or cardiac anomalies in patients with MDAs, could also affect their conception, fertility, modes of delivery, and obstetrical outcomes [7].

The diagnosis of MDAs remains challenging in most cases. Although hysterosalpingogram is useful in diagnosing a unicornuate uterus, it does not help in detecting a noncommunicating horn. Two-dimensional (2D) ultrasound is the initial imaging modality of choice because it is widely available, noninvasive, relatively inexpensive, and able to provide information about other relevant nonuterine structures such as ovaries, kidneys, and pelvic mass. It can also provide information on the subsequent imaging modality or modalities that should be chosen for definitive diagnosis. In contrast, threedimensional (3D) reconstructed images provide more detailed information and often prevent the need for additional imaging $[8,9]$. Historically, magnetic resonance imaging has been the gold standard for the diagnosis of reproductive tract anomalies [10]. However, magnetic resonance imaging is reserved for cases only when $2 \mathrm{D}$ or 3D ultrasound findings are limited and a definitive diagnosis is required to make decisions regarding patient care. Additional investigations include an intravenous pyelogram or renal ultrasonography to detect any horseshoe kidney, ipsilateral renal agenesis, or pelvic kidney. Laparoscopy is rarely indicated in the investigation of MDAs.
Among the various types of MDAs, the unicornuate uterus is associated with the poorest fetal survival. Even if the pregnancy is healthy, the obstetric performance is universally poor in this group. Common obstetrical complications include malpresentation, intrauterine growth retardation, and preterm birth. These reproductive problems are attributed to abnormal uterine vasculature and diminished myometrial mass of the unicornuate uterus [11]. As a result, cesarean delivery rates are high among this group of patients.

In view of these complications, it is crucial for female patients with multiple congenital anomalies to receive an early referral to exclude MDAs. A thorough assessment is compulsory during the initial pregnancy checkup to assess the severity of the condition. Early recognition and categorization of the condition allow appropriate management during the antepartum, intrapartum, and postpartum periods to ensure a good outcome for mother-baby dyad care.

However, our patient, even though she underwent regular antenatal review at the local health center, was referred only much later to our tertiary-level center for further evaluation. Her old admission records regarding her childhood conditions were not available. On the basis of limited records, her clinical findings were suggestive of vertebral, anorectal, tracheoesophageal, renal, and limb associations. Hence, the possibility of an underlying MDA should be highly suspected from the beginning. The MDA was proved intraoperatively with the presence of unicornuate uterus with a rudimentary horn and communicating uterine horn. This condition is frequently associated with ipsilateral renal and ureter agenesis [12]. However, it was absent in our patient.

\section{Conclusion}

The majority of MDAs remain undiagnosed due to a lack of clinical suspicion and the absence of pathognomonic clinical and radiological characteristics. Hence, knowledge of its existence and the role of antenatal radiological investigations is vital to prevent the wide range of gynecological and obstetrical complications. As illustrated in our patient's case, the presence of multiple congenital abnormalities and a history of infertility in a pregnant woman should warrant the exclusion of MDAs from the beginning. Early detection of MDAs will facilitate the most appropriate delivery plan to ensure the best maternal and neonatal outcomes.

\section{Abbreviation \\ MDA: Müllerian duct anomaly}

Acknowledgements

The authors thank the patient for her consent to publish her case. 


\section{Authors' contributions}

EIEH, NLNAZ, and KMN take full responsibility for the information presented. The authors agree that they have full confidence in the accuracy and integrity of the work. The authors contributed at all levels, from data collection to the writing and submission of the manuscript. All authors read and approved the final manuscript.

\section{Funding}

No funding was received for the publication of this report.

\section{Availability of data and materials}

The patient's information and medical records used for the case report are available from the corresponding author upon request.

\section{Ethics approval and consent to participate}

This study was conducted in accordance with the fundamental principles of the Declaration of Helsinki.

\section{Consent for publication}

Written informed consent was obtained from the patient for publication of this case report and any accompanying images. A copy of the written consent is available for review by the Editor-in-Chief of this journal.

\section{Competing interests}

The authors declare that they have no competing interests. The authors have no association with commercial entities that could provide support for the work reported in this report or with entities that could be viewed as having a general interest in the area of the submitted manuscript. The authors have no association with financial or nonfinancial organizations.

Received: 27 May 2020 Accepted: 17 August 2020

Published online: 02 November 2020

\section{References}

1. Chan YY, Jayaprakasan K, Zamora J, Thornton JG, Raine-Fenning N, Coomarasamy A. The prevalence of congenital uterine anomalies in unselected and high-risk populations: a systematic review. Hum Reprod Update. 2011;17(6):761-71.

2. American Fertility Society. The American Fertility Society classifications of adnexal adhesions, distal tubal occlusion, tubal occlusion secondary to tubal ligation, tubal pregnancies, müllerian anomalies and intrauterine adhesions. Fertil Steril. 1988:49(6):944-55.

3. Grimbizis GF, Camus M, Tarlatzis BC, et al. Clinical implications of uterine malformations and hysteroscopic treatment results. Hum Reprod Update. 2001;7:161-74.

4. Troiano RN, McCarthy SM. Mullerian duct anomalies: imaging and clinical issues. Radiology. 2004;233(1):19-34.

5. Li S, Qayyum A, Coakley FV, Hricak H. Association of renal agenesis and mullerian duct anomalies. J Comput Assist Tomogr. 2000;24(6):829-34.

6. Oppelt P, Renner SP, Kellermann A, et al. Clinical aspects of MayerRokitansky-Kuester-Hauser syndrome: recommendations for clinical diagnosis and staging. Hum Reprod. 2006;21(3):792-7.

7. Vilanova-Sanchez A, McCracken K, Halleran DR, Wood RJ, Reck-Burneo CA, Levitt MA, Hewitt G. Obstetrical outcomes in adult patients born with complex anorectal malformations and cloacal anomalies: a literature review. J Pediatr Adolesc Gynecol. 2019:32(1):7-14.

8. Shatzkes DR, Haller JO, Velcek FT. Imaging of uterovaginal anomalies in the pediatric patient. Urol Radiol. 1991;13:58-66.

9. Raga F, Bonilla-Musoles F, Blanes J, Osborne NG. Congenital Müllerian anomalies: diagnostic accuracy of three-dimensional ultrasound. Fertil Steril. 1996;65:523-8.

10. Bakri YN, al-Sugair A, Hugosson C. Bicornuate nonfused rudimentary uterine horns with functioning endometria and complete cervical-vaginal agenesis: magnetic resonance diagnosis. Fertil Steril. 1992;58:620-1.

11. Lin PC. Reproductive outcomes in women with uterine anomalies. J Womens Health (Larchmt). 2004;13(1):33-9.

12. Buttram VC Jr, Gibbons WE. Müllerian anomalies: a proposed classification (an analysis of 144 cases). Fertil Steril. 1979;32(1):40-6.

\section{Publisher's Note}

Springer Nature remains neutral with regard to jurisdictional claims in published maps and institutional affiliations.

Ready to submit your research? Choose BMC and benefit from:

- fast, convenient online submission

- thorough peer review by experienced researchers in your field

- rapid publication on acceptance

- support for research data, including large and complex data types

- gold Open Access which fosters wider collaboration and increased citations

- maximum visibility for your research: over $100 \mathrm{M}$ website views per year

At $\mathrm{BMC}$, research is always in progress.

Learn more biomedcentral.com/submissions 